\title{
L'héroïque et les longs poèmes de Guy Le Fèvre de La Boderie
}

Jean Céard

\section{(2) OpenEdition}

\section{Journals}

Édition électronique

URL : https://journals.openedition.org/crm/1982

DOI : $10.4000 / \mathrm{crm} .1982$

ISSN : 1955-2424

Éditeur

Honoré Champion

Édition imprimée

Date de publication : 15 avril 2004

Pagination : 25-35

ISSN : $1272-9752$

Référence électronique

Jean Céard, «L'héroïque et les longs poèmes de Guy Le Fèvre de La Boderie », Cahiers de recherches médiévales [En ligne], 11 spécial | 2004, mis en ligne le 18 octobre 2007, consulté le 15 décembre 2022. URL : http://journals.openedition.org/crm/1982 ; DOI : https://doi.org/10.4000/crm.1982

Ce document a été généré automatiquement le 15 décembre 2022.

Tous droits réservés 


\title{
L'héroïque et les longs poèmes de Guy Le Fèvre de La Boderie
}

\author{
Jean Céard
}

1 C'est une question paradoxale que soulève ce titre. Il est courant, en effet, de ranger les longs poèmes de Guy Le Fèvre de La Boderie parmi les poèmes qu'on appelle conventionnellement scientifiques ou encyclopédiques. Pourtant la tradition du long poème ressortit à la poésie épique, ou, si l'on préfère, à la poésie héroïque, qu'on l'appelle simplement «long poëme", comme le fait Du Bellay (Deffence, II, 5), ou que l'on précise, comme Peletier (Art poétique, II, 8), « CEuvre héroïque ». Après l'Encyclie des secrets de l'Eternité, publiée en 1571, La Boderie donne, en 1578, La Galliade, ou De la Revolution des Arts et Sciences, dont la seconde édition parait en 1582. Ces œuvres se trouvent ainsi être étroitement contemporaines des plus célèbres Sepmaines de Du Bartas. Or, celui-ci, dans son Brief Advertissement bien connu de 1584, écrit: "Ma seconde Sepmaine n'est (aussi peu que la premiere) un œuvre purement Epique, ou Heroïque, ains en partie Heroïque, en partie Panegirique, en partie Prophetique, en partie Didascalique $»^{1}$. On se prend à regretter que cette définition ne soit pas de La Boderie, tant elle paraît convenir à ses longs poèmes !

2 Mais La Boderie ne nous laisse malheureusement pas la ressource de nous autoriser de son silence pour appliquer à son œuvre la définition de DuBartas. Dans l'«Advertissement aux Lecteurs" de La Galliade, il récuse explicitement le terme d'« héroïque » :

Afin, Lecteurs debonnaires, que vous ne vous esgariez aux replis de cest Æuvre, ainsi que dans un Labyrinthe, j'ay bien voulu vous advertir, que le fil d'iceluy n'est pas tissu, tramé, ny ourdy à la façon du Poëme Heroïque, duquel la toile est pour la pluspart tissue de fables, et contes plaisants, et qui se propose plustost pour son but et fin la delectation, et la vraysemblance, que non pas l'utilité et la verité. Or l'ay-je voulu nommé Galliade, non pour imiter Iliades, Eneides, ny autres tels Poëmes et inventions empruntees des fables moisies des Grecs, ou des vieux Romans : mais parce que je me suis essayé d'y traiter de la Revolution des Arts et Sciences ${ }^{2}$.

Cette déclaration appelle deux observations, l'une sur les raisons pour lesquelles l'auteur récuse le modèle du poème héroïque, l'autre sur le titre qu'il choisit de donner 
à son œuvre. Pour commencer par la seconde, il faut remarquer que certes La Boderie précise que le mot de Galliade renvoie au terme hébreu de Galal: "J'emprunte l'etymologie, et deduction de Galliade du verbe Hebrieu Galal, qui signifie Reployer et retourner ", terme qu'il applique à cette révolution des arts et sciences qui les a fait, après qu'ils ont circulé "presque par tout le Rond de la Terre », retourner " en nostre Gaule». Le terme est certes adroitement choisi, qui implique à la fois l'idée de révolution et celle du lieu, bien nommé, où le cercle se boucle. Il n'empêche que la forme, retenue par La Boderie, de Galliade, par son suffixe caractéristique, rappelle si évidemment les noms des poèmes épiques que l'auteur se sent tenu de souligner que sa Galliade ne prend pas modèle sur eux. Pourquoi ne l'avoir pas simplement évité ? On dirait que le modèle de l'Iliade et de l'Enéide, pour citer les deux illustres exemples mentionnés par La Boderie, demeure à l'arrière-plan d'une œuvre qui s'obstine à le récuser.

Si La Boderie le rejette, ses raisons sont claires, et c'est la première observation appelée par sa déclaration : la toile du poème héroïque « est pour la pluspart tissue de fables ", elle n'est ourdie que d'« inventions empruntees des fables moisies des Grecs, ou des vieux Romans ». Denis Bjaï a bien noté, en recueillant diverses attestations, que l'expression de "fable moisie ", due sans doute d'abord à Du Bellay, est devenue «le signe de reconnaissance du poète chrétien, tout particulièrement dans les années $1570 »^{3}$. J'ajouterai que la mention des «vieux Romans» semble bien renvoyer à la proposition de Du Bellay, qui, dans la Deffence, II, 5, invitait à choisir « un de ces beaux vieulx romans Francoys, comme un Lancelot, un Tristan, ou autres " et à en faire "renaitre au monde une admirable Iliade et laborieuse Eneide», ou à l'exhortation de Peletier (Art poétique, II, 8), qui, trouvant «noz rommans bien invantiz», assurait le " poëte heroique » qu'il en ferait utilement son profit. Tout cela n'est, pour La Boderie, que «bourdes », car c'est de ce mot qu'il se plaît à désigner les fables, et notamment les fables antiques. On n'aurait jamais fait si l'on voulait relever toutes les occurrences de cette idée, qui court dans toute l'œuvre de La Boderie. En voici un simple exemple, emprunté à l'Encyclie, qui célèbre, dans une longue pièce liminaire à valeur programmatique,

Ceste Lyre où jamais les fables mensongeres

De ces faus Dieus Gregeois je ne veux fredonner,

Ni les vaines chansons profanes et legeres

De l'impudique Amour y faire resonner,

Ni plus renouveler les bourdes estrangeres 4 .

5 L'hostilité de La Boderie à la poésie impudique et au mensonge de la fable réunit dans un même combat ces deux objets, au nom de la vérité, nécessairement haute et chaste. Car «utilité » et "verité » vont de pair, et s'opposent à la «délectation » et à la «vraisemblance». On n'écrit pas pour charmer, mais pour instruire; et l'on ne peut instruire que de la "vérité " et par la "vérité ", dont la vraisemblance est une condamnable contrefaçon. Il sait que l'une de ses sources, Annius de Viterbe, est déjà très contestée de son temps, mais il répond que mille autres témoignages confirment ce qu'il appelle «nostre cause », et il tient, dit-il, à le préciser «afin qu'il ne semble que poëtisant j'aye seulement voulu conter des bourdes et plaisanteries » (p. 155).

Ce choix entraîne avec lui le rejet de la poésie héroïque, puisqu'aux yeux de La Boderie, elle est inséparable de la recherche du plaisir et de la pratique de la simple vraisemblance. Est-ce à dire qu'il rompt totalement avec elle ? Il semble bien que, s'il n'a nulle sympathie pour le mot, il ne se détourne pas tout à fait de la chose. Le titre 
même de Galliade, si épique d'allure, mais tout autrement justifié, en est le premier signe.

7 Certes La Boderie ne se résout pas à écrire un poème épique (ou héroïque, car de son temps les deux mots semblent être étroitement synonymes). Il promet à son maitre, le duc d'Alençon, qui se prénommait François-Hercule, de chanter ses hauts faits s'il décide d'aller reconquérir Jérusalem :

Que s'il te vient à gré d'écouter ma chanson, Desor je te promets, ô grand Duc d'Allençon, Qu'à ton retour de là en triomfe et en pompe Je feray retentir tes vertus dans la trompe.

Et s'il te plaist un jour mes vers favoriser, Et dessous ta grandeur ma Muse authoriser, Je chanteray si haut ta grand' Herculeide Qu'elle suivra de pres l'Iliade et l'Eneide ${ }^{5}$.

En 1578, dans un sonnet liminaire de La Galliade, il renouvelle sa promesse :

Et l'Alcide rangé

Dans le Char triomfal de son beau nom changé,

Apres la Galliade orra l'Herculeïde ${ }^{6}$.

Je ne suis pas sûr que La Boderie ait négligé cette promesse parce que le duc d'Alençon ne lui a pas donné matière à la satisfaire. Je crois plutôt qu'il n'a guère de goût pour le genre du poème héroïque. Avant d'en chercher les raisons, il est bon de remarquer en quels termes il définit les écrivains dont La Galliade célèbre la contribution, puisque ce long poème comporte, en son cinquième livre, une sorte d'histoire de la poésie, où il recense notamment ce que les Latins lui ont apporté. Pour Silius Italicus, il emploie deux fois le terme d'héroïque :

Puis l'Italic Sillie en héroïques carmes

Luy feist la trompe enfler des héroïques armes?

10 Mais cet emploi est exceptionnel. La Boderie omet le terme pour parler de Lucain et de Stace, non sans dire toutefois qu'ils chantèrent

Les combats Martiaux, et vaillance des Preux ${ }^{8}$.

11 Il est d'autant plus frappant de constater que son éloge de Virgile, qu'il met au plus haut, accorde dix vers au poète des Bucoliques et surtout des Géorgiques, mais n'en concède que deux à l'Enéide, et sans le mot d'héroïque :

Mais le fort vent s'empoupe en ses vaisseaux ramez

Quand les armes il chante, et les hommes armez ${ }^{9}$.

Même omission dans sa célébration d'Homère. Certes le souci de la variété et de la copia peut l'expliquer; il n'empêche que La Boderie n'est pas avare de termes génériques (lyrique, élégiaque, tragique, satirique, etc.).

13 Vient-il à la poésie moderne ? Il loue certes Ronsard, mais c'est à sa veine pindarique et lyrique qu'il s'intéresse; de la Franciade, nulles nouvelles. Pour Marot, il signale en passant sa traduction d'Ovide, de «la Metamorphose héroïque et nombreuse » ${ }^{10}$. Un seul poète est caractérisé par sa "veine héroïque", mais il s'agit d'Héroët, et La Boderie, qui aime à motiver les noms propres, ne pouvait manquer la paronomase, «Le docte Héroët à la veine heroïque » (V, 961), comme Du Bellay a un «nom beau » $(V, 1019)$, et comme Muret est un poète « aux vers murs » $(V, 1074)$. On pourra objecter que La Galliade n'est pas tenue de tout dire, que ce n'est qu'une évocation, comme en pointillés, de la vaste histoire de la poésie. Il faut donc noter que l'Encyclie, qui n'a pas le 
même objet, se place sous l'invocation de Ronsard, mais le Ronsard des Odes et des Hymnes:

Le Pindare François sur la Lire à sept cordes

Premier a r'animé les tons melodieus

Des Grecs et des Romains, et en Hymnes et Odes

Celebré les vertus des hommes et des Dieus ${ }^{11}$.

14 Autre omission de la Franciade, qui certes n'est pas alors publiée, mais dont, à cette date, tout le monde sait que Ronsard y travaille, et qui paraîtra l'année suivante. C'est au point que, si La Boderie n'était un homme sans malice, on aurait le droit de chercher à savoir pourquoi, dans sa revue relativement brève des poètes latins, il choisit de citer Lucain, Stace et Silius Italicus, et pour ce dernier, on l'a dit, non sans un emploi redoublé et voyant d'héroïque. J'en viens à me demander si ce n'est pas parce que ces poètes ont pris pour sujet l'histoire véritable, même si tous trois, mais surtout Silius Italicus, n'en ont pas saisi le dessein profond. On y verrait volontiers une réplique à Ronsard, si les propos que je vais citer n'appartenaient à la préface posthume de la Franciade. Non seulement Ronsard y met ces poètes au nombre de ceux « ausquels on ne parle jamais que deus ou trois fois en la vie » (Lm, XVIII, 351), mais surtout il leur fait grief d'avoir choisi un sujet historique: «Les autres vieils Poetes Romains comme Lucain et Silius Italicus, ont couvert l'histoire du manteau de Poesie : ils eussent mieux fait à mon advis, en quelques endroits, d'escrire en prose » (Lm, XVIII, 338-339).

Quel est le statut des personnages que célèbre La Boderie ? Ne peut-on les considérer comme les héros de cette poésie vraie ? À vrai dire, La Boderie ne leur donne jamais ce nom. Parmi les personnages qui défilent dans son œuvre, les uns, vite mentionnés, sont de grands guerriers, à qui il donne volontiers le nom de « Preux » : Homère a chanté les Preux $(V, 569)$; une apostrophe au duc d'Alençon commence par ces vers :

o Alcide l'Heureux, ô Prince de renom,

Qui du premier des Preux as le tiltre et le nom ${ }^{12}$.

16 Il peut même une fois leur donner le nom de "héros", comme dans cet éloge des Bardes gaulois,

Qui les premiers ont joint les vers à la Musique

Que du los des Herôs ils ont faite héroïque ${ }^{13}$.

17 Mais cet emploi est, sauf erreur, unique, et, de toute façon, l'intérêt de La Boderie ne va nullement à ces personnages, qui ne sont ici mentionnés que pour les éloges que les bardes leur ont décernés. Les autres, infiniment plus considérés, sont tous ceux qui ont contribué à la "révolution des arts et sciences ». Ils sont çà et là désignés comme des "hommes de marque», des "personnages doctes et signalés", des «hommes remarquables », d'« excellents esprits $»^{14}$. Et La Boderie fait de leur célébration son sujet, en les opposant aux guerriers. Un sonnet liminaire de La Galliade, justement noté par D. Bjaii ${ }^{15}$, le dit en termes fort clairs au dédicataire, le duc d'Alençon :

Vostre Gaule a nourry une troupe nombreuse

De celestes Esprits, lesquels de toutes parts

Nous y ont ramené la riviere des Arts

Qui surgeonna jadis de sa fonteine ombreuse.

D'eux icy j'ay sonné dessus ma Lyre creuse

La gloire et les honneurs : autres diront de Mars

Les beaux faits, et le prix des Gaulois Estendars,

Qui auront plus que moy la Muse et main heureuse :

De moy je suis content pour ma part d'avoir pris

La charge d'illustrer les illustres Espits, 
De fables n'ourdissant une longue Iliade :

Mais de juste loyer et los de verité

Ornant mon Peuple, et Gent, comme il a merité,

Et des Gaules, Gaulois, chantant la Galliade ${ }^{16}$.

Tous les motifs chers à La Boderie sont ici réunis : l'opposition des armes et des lettres, si nette que les hommes de guerre n'apparaissent qu'à travers «les beaux faits de Mars » et les «Gaulois estendars », tandis que les hommes de lettres (j'entends par là, d'un mot, ceux qui ont pratiqué les sciences et les arts) sont désignés comme «de celestes Esprits » et d'« illustres Esprits »; l'opposition fondamentale de la fable et de la vérité ; mais aussi, comme le souligne la rime de l'Iliade et de la Galliade, l'affirmation que le long poème que l'on va lire a la dignité, la hauteur et peut-être la qualité d'un poème héroïque, sans en avoir les insuffisances et les défauts. Certes La Boderie, selon un motif bien connu, assure avoir retenu ce sujet parce qu'il se sent incapable de chanter «de Mars/Les beaux faits ", mais ne doutons pas qu'il ne soit convaincu de faire bien mieux en faisant autrement.

À ces « celestes Esprits ", à ces «illustres Esprits ", il ne manque que le nom de héros pour que le poème qui leur est consacré soit qualifié d'héroïque. Ce pas, pourtant, La Boderie ne le franchit point. On peut se demander si ce n'est pas sa vision de l'histoire qui l'en dissuade. Tous ces grands hommes n'ont été des initiateurs et des inventeurs que dans la mesure où ils ont adhéré de tout leur cœur au dessein de Dieu et ont voulu contribuer à sa réalisation. Ils méritent certes d'être célébrés, en ce qu'ils ont les premiers entendu l'appel divin et y ont répondu. C'est ainsi que Misraim-Osiris, fils de Cham, qui reçut en partage l'Égypte, sut en profiter pour inventer l'agriculture, et doit donc être salué comme celui

Qui le premier de grains les hommes nourrissant

Avec sa femme et seur Isis la vagabonde

Se feist pour sa bonté honorer par le monde.

La Boderie continue :

La premiere Charue il feist d'ouvriere main,

Premier du soc fendit de la terre le sein,

Premier meist la semence en terre non experte,

Et aux arbres cueillit la pomme meure et verte :

Premier la tendre vigne aux eschallats coupla,

Et de serpe d'acier premier il l'escoupla ${ }^{17}$.

Voilà en quoi Misraim-Osiris a droit à notre mémoire, comme Magus qui, «le premier », inventa l'architecture (II, 93), comme Bardus à qui la poésie «doit son commencement » (IV, 5). Il en va de même de tous les autres inventeurs, qui réalisent le dessein divin, lui donnent forme, l'actualisent. Je ne dirais pas, avec François Roudaut : "C'est pourquoi ceux qui instruisent les hommes apparaissent comme des héros", puisque La Boderie me semble éviter ce mot, mais je ne puis qu'adhérer à son commentaire : « Moins que de le découvrir, l'homme doit accomplir le monde, c'est-àdire imiter l'architecte divin en faisant passer dans la réalité ce qui est dans "l'entendement divin".» Je retiendrai aussi, de François Roudaut, cette juste remarque: "Malgré la présence d'hommes providentiels, l'aventure humaine est marquée dans $L a$ Galliade par des mouvements de peuples $"^{18}$. En ce sens, les hommes providentiels ne sont que les guides, les conducteurs des peuples, ou même ceux dont le nom les résume : ainsi Misraim, qui est aussi le nom de l'Égypte. On ne saurait pourtant omettre de les signaler parce que c'est à travers eux que prend sens la formule du livre 
des Proverbes qui sert d'épigraphe à La Galliade, formule d'abord inscrite en hébreu puis traduite par ce distique :

C'est la gloire de Dieu la parole cacher,

C'est la gloire des Rois la parole chercher.

Les rois et, plus généralement, les grands hommes sont ceux qui, cherchant la parole de Dieu, ont progressivement contribué à la dévoiler. Tel est le sens de l'histoire, qui est bien, par eux, révélation progressive. Le maître de La Boderie, Guillaume Postel, commentant le texte de l'épigraphe, l'indique fortement: « Ce fut toujours jusques ici, où il faut que toutes choses soient restaurées, la gloire de Dieu de cacher sa parole, pour qu'en chacun des temps, il y eut toujours progrès $"{ }^{19}$. Humble mais très nécessaire mission des grands hommes, qui leur mérite notre respect et notre admiration. Et c'est pourquoi, résumant son œuvre dans une pièce des Divers Meslanges poétiques, La Boderie se contente de caractériser ainsi sa Galliade :

J'ay recueilly des vieux Autheurs estranges

De noz Gaulois les antiques louanges,

Et illustré noz hommes dessus tous

Qui faict renaistre ont les arts entre nous ${ }^{20}$.

Il faut maintenant se demander pourquoi La Boderie, flirtant, si j'ose dire, avec la poésie héroïque, ne se décide pas à la reconnaître pour son modèle, fût-ce en le purifiant, c'est-à-dire en l'habituant à s'abstenir des «fables moisies » et à dire vrai. La question se pose d'autant plus que Guillaume Postel émit des réserves sur la forme choisie par son disciple; F. Roudaut et D. Bjaï ont opportunément mentionné ce jugement, qu'il faut rappeler : «[...] nous ou Celtes ou Galathes ou Gauloys jadis premier que les Hiberes surnommez, et qui principalement avant que les Grecs, plus de 2000 ans eusmes et characteres, et langue et escripture propre, come tres veritablement en la Galliade de Faber se prouve, combien qu'en vers, à mode poetique subject à discussion $»^{21}$. «Veritablement »: Postel donne quitus à La Boderie qui n'a pas versé dans le travers fabuleux de la poésie héroïque, tel que le stigmatise le poète lui-même. Mais c'est le choix même des "vers, à mode poétique » que conteste Postel. Cette réserve et la pratique contraire de La Boderie soulèvent ainsi deux questions : pourquoi La Boderie se refuse-t-il à pratiquer l'écriture épique (ou héroïque) ? Pourquoi choisit-il cependant la forme poétique?

Sur le premier point, la réponse semble bien découler de sa vision de l'histoire. Si les hommes, si grands soient-ils, sont d'abord les serviteurs du dessein de Dieu, il ne convient pas de leur conférer la solitude du héros épique. Leur action prend sens dans une continuité, même si, de telle bénéfique invention, ils sont les initiateurs. Cette vision me semble, en outre, s'accorder à la veine de La Boderie, qui répugne aux lenteurs du récit épique, avec ses préparations, ses descriptions, ses transitions, d'un mot ses contraintes narratives nécessaires pour en ourdir la toile. On le voit bien quand, dans une longue pièce des Hymnes ecclesiastiques, dédiée à Ronsard et intitulée "Hymne à Jesus-Christ nostre Seigneur de ses victoires et triomfes ", il relate certains moments de cette glorieuse aventure. Veut-il évoquer la vie de Jacques le Majeur? Il écrit :

Aux Solimes encor le grand Jacques parla,

Et du glaive pointu de sa foy et faconde

Poignit, et puis plongea dedans la sacrée onde

Philete, et Hermogene, outre mesure enflez

De leurs magiques arts par les demons soufflez : 
Puis passe un grand traject en l'Espagne guerriere

Qui l'admire et cherit : là de la fin derniere

Du Levant élongné, et des mercs et confins

Des Ethiopes noirs, les peuples Abissins

$\mathrm{Y}$ viennent voyager, car par la mer enflée

Une barque du vent de l'Eternel soufflée

Y apporta son corps en Gallice arrivant,

Où son tombeau sacré par miracle est vivant ${ }^{22}$.

dont la vie toute retirée n'est évidemment pas fertile en événements, il n'est ni plus ni moins prolixe. Ainsi de l'ermite Paul :

Plus se pleut le bon Paul qui solitaire habite

Dans le vague hermitage estant premier Hermite

Aux dattes qu'il cueilloit du Palmier ombrageux

Et à l'habit tissu de fueillards nuageux,

A l'onde pure et claire au creux des mains puisee,

Qu'aux delices des Roys et viande prisee,

Qu'au doux vin de Phalerne en coupe d'or riant

Ou à la laine teinte en pourpre d'Orient ${ }^{25}$.

est donc pas la plus ou moins abondance de la matière qui guide La Boderie. Et ce n'est pas non plus la plus ou moins grande célébrité des personnages signalés et des aventures relatées qui retient ou excite sa plume. Si, par exemple, Magellan est bien connu, il n'est pas sûr que ses lecteurs sachent dans son détail son action à Subuth, dans l'archipel des Moluques. Voici la relation de La Boderie, faisant au Christ hommage de l'aventure de Magellan :

Jà l'Isle de Subuth ayant sa Loy changée

Pour la tienne embrasser, a dessouz soy rangée

Toute autre Isle voisine, et leurs peuples soumis,

Depuis que Magellan ton Nocher et commis

Leur eut persuadé ta Loy tant douce prendre,

Quand le Neveu du Roy tout sain il te plest rendre,

Ton Magellan qui a tout le monde couru,

Et en bien combatant a pour toy encouru

Une mort honorable, et à jamais heureuse,

Apres t'avoir gaigné ceste Isle plantureuse ${ }^{26}$.

Il faut chanter Magellan, qui, « Nocher et commis » de Dieu, l'a remarquablement servi; mais, si l'on veut savoir de quelle merveilleuse guérison il a été l'occasion ou quelle fut sa mort, il vaut mieux aller consulter les historiens des Découvertes. Nous ne lirons pas une Magellanéide, même si, assurément, tous les ingrédients épiques pourraient y être mis en œuvre et rajeunir et christianiser l'Odyssée. Au reste, si nombreux soient les détails que l'on pourrait puiser dans les œuvres des historiens, il faudrait les habiller à la mode épique. C'est à quoi répugne La Boderie: agir ainsi, ce serait commencer à farder l'histoire, donc à travestir la vérité. 
29 Mais renoncer à ces oripeaux, ce n'est pas renoncer à la poésie. La Boderie tient la poésie pour la forme la plus adéquate à la célébration de Dieu. Il est significatif de constater l'insistance qu'il met à mentionner la forme poétique dans la traduction de l'hymne d'Hermès Trismégiste qu'il insère dans La Galliade. L'original dit seulement: "Que ton verbe par moi te loue, par moi le monde reçoit ce sacrifice de paroles, mes forces crient, chantent le tout, et font ta volonté. Que ta volonté passe de toi au tout ; ce sacrifice de paroles, reçois-le de tous. " La Boderie traduit :

Par moy t'aille louant ton Verbe tressacré,

Par moy reçoit le monde un sacrifice à gré

De paroles et vers, haut mes forces s'escrient,

Elles chantent le Tout, et le Tout elles crient,

Et font ta volonté, où toute leur fin tend :

Ta sainte volonté de toy en tout s'estend :

Reçoy donques de tous, de tous païs estranges

Sacrifice de vers, offrande de louanges ${ }^{27}$.

Ce dernier vers définit clairement l'orientation générale de la poésie de La Boderie : la forme poétique qu'il affectionne est celle du lyrisme de célébration. Ses vrais modèles, constamment allégués, ce sont David et Pindare, et le poète français qu'il élève au plus haut est, on l'a dit, le Ronsard des Odes et des Hymnes.

31 La fin dernière de cette poésie, c'est d'offrir le monde à Dieu. Un Dieu qui est lui-même poète, et dont le monde est le poème, comme l'indique justement François Roudaut ${ }^{28}$. On connaît la formule célèbre du livre de la Sagesse : Omnia in mensura, et numero, et pondere disposuisti $i^{29}$. Elle est sans cesse présente à l'esprit de La Boderie, comme pourrait le montrer l'Encyclie des secrets de l'Eternité. Pour nous en tenir à La Galliade, il est frappant de voir que, parmi les sciences et arts, l'attention de La Boderie se tourne surtout vers l'architecture, la musique et la poésie. Puisque le monde est mesure, nombre et poids, il ne peut être dit convenablement que par une œuvre qui s'en inspire. Une œuvre qui ne peut pleinement réaliser cette consonnance avec le monde que si elle est elle-même inspirée. La Boderie l'indique en décrivant le plan de son poème et en notant qu'il a consacré le dernier livre à la poésie, «laquelle, dit-il, bien que je l'aye mise entre les Arts et Sciences, semble toutesfois estre plustost une saincte fureur et elevation d'esprit, que non pas une doctrine acquise par industrie et puissance humaine $»^{30}$.

On ne s'étonnera pas que La Boderie note plusieurs fois combien le culte des faux dieux s'est insinué dans la poésie, combien leurs fables et leurs bourdes l'ont souillée. Heureux le temps où l'on ne peignait point

de fables et mensonges

Semblables proprement des malades aux songes

Les ouvrages divins, ains sans art, et sans fard

On celebroit l'ouvrier duquel l'ouvrage part ${ }^{31}$.

La révolution des sciences et des arts, gage d'une restauration, nous fait espérer une retour de cette simple et vraie pureté. Ne la retardons pas en nous contentant de sacrifier à la poésie héroïque, fût-ce au bénéfice du grand duc d'Alençon. 


\section{NOTES}

1. Texte établi et commenté par D. Bjaï, « La Sepmaine, "œuvre... en partie heroïque”, ou les enjeux d'un Brief advertissement », «La Sepmaine de G. du Bartas », Cahiers Textuel $\mathrm{n}^{\circ} 13,1993$, p. 57.

2. Guy Le Fèvre de La Boderie, La Galliade, éd. Roudaut, Paris, Klincksieck, 1993, p. 153. Passage commenté par D. Bjaï, La Franciade sur le métier. Ronsard et la pratique du poème héroïque, Genève, Droz, 2001, pp. 440-443.

3. D. Bjaï, op. cit., p. 441.

4. Guy Le Fèvre de La Boderie, L'Encyclie des secrets de l'Eternité, Anvers, Ch. Plantin, [1571], p. 24. D'autres occurrences sont mentionnées dans mon art. «Christianisme et paganisme dans les Hymnes Ecclesiastiques de Guy Le Fèvre de La Boderie », Cité des hommes, cité de Dieu, Genève, Droz, 2003, p. 381-392.

5. Encyclie, p. 225. Partiellement cité par D. Bjaï, op. cit., p. 437.

6. Galliade, p. 159.

7. Galliade, V, 791-792, p. 543.

8. Galliade, V, 782, p. 543.

9. Galliade, V, 743-758, pp. 541-542.

10. Galliade, V, 954, p. 549.

11. Encyclie, p. 25.

12. Galliade, IV, 1257-1258, p. 486.

13. Galliade, IV, 1779-1780, p. 507.

14. Galliade, "Advertissement aux lecteurs », p. 155, 156, 157.

15. D. Bjaï, op. cit., p. 440.

16. Galliade, p. lim., Sonnet 7, p. 146.

17. Galliade, I, 853-862.

18. François Roudaut, Le point centrique, Paris, Klincksieck, 1992, p. 70.

19. Cité par Roudaut, ibid., p. 69.

20. Divers Meslanges poétiques, éd. Rosanna Gorris, Genève, Droz, 1993, p. 169. Cité par

D. Bjaï, p. 438.

21. Fr. Roudaut, éd. Galliade, p. 93 ; D. Bjaï, p. 438.

22. Les Hymnes Ecclesiastiques, Paris, R. Le Mangnier, 1582, f. 200 [pièce 2.2, vv. 864-876].

23. Il faut aller relire au moins la Légende dorée pour comprendre la mention de Philète et Hermogène.

24. Ibid., f. 201 [vv. 919-924].

25. Ibid., f. 198 [vv. 781-788].

26. Ibid., f. 204 [vv. 1105-1114]. Voir Maximilianus Transsylvanus, Epistola de admirabili et nouissima Hispanorum in Orientem nauigatione, Rome, Minitius Caluus, $1524, \mathrm{f}^{\circ} \mathrm{C}$ iv v ${ }^{\circ}-$ Do $\mathrm{r}^{\circ}$ : Arrivés à l'île de Subuth, dans les Moluques, Magellan et ses compagnons rencontrent le roi. «Conspicit Magellanus in domo Regali quendam aegrum et iam prorsus extenuatum. Scrutatur quis nam hic esset, et quo morbi genere torqueretur. Intelligit nepotem illius reguli et iam Biennio vehementi febre laborasse. Iubet eum bono animo esse, si modo se Christo deuoueret, protinus salutem et pristinam valetudinem recuperaturum. Indus conditionem accipit, cruceque adorata, baptismum capit. Postridie se incolumen affirmat, surgit ex lectulo, ambulat, cibum sumit ut reliqui. » 27. Galliade, IV, 1681-1688. Le texte du Pimandre est cité p. 502. 
28. F. Roudaut, éd. Galliade, Introduction, p. 95.

29. Sg, XI, 21.

30. Galliade, p. 157.

31. Galliade, V, 7-10, p. 509. Allusion aux célèbres aegri somnia, d'Horace, A.P., 7.

\section{AUTEUR}

JEAN CÉARD

Université de Paris X-Nanterre 